\title{
ESTADISTICA
}

\section{Núcleos de agrupación}

\section{de la población española}

Al reanudar en estas páginas nuestra colaboración estadística, creemos un deber dedicar cariñoso recuerdo al que en vida fué gran amigo y compañero, Javier Ruiz Almansa (q. e. p. d.), cuyas huellas, tan dignas de elogiosa memoria, aún palpitan en esta Revista y en varias monografías del Instituto de Estudios de Administración Local.

Realizado en fin de diciembre de 1950 , por el Instituto Nacional de Estadistica, el Censo decenal de la población española y en plena tarea de elaboración las múltiples clasificaciones a que da lugar, acaban de conocerse las cifras definitivas globales.

La población de derecho, formada, como se sabe, por los habitantes residentes, ya estén presentes o ausentes del Municipio, alcanza en el último censo la cifra de 28.039.112, de los cuales, 7.458.252 viven en capitales y su distribución por Provincias y capitales figura en el cuadro I.

Por primera vez, además de la acostumbrada clasificación de los habitantes según su distribución en Municipios con arreglo a unos limites marcados, se ha llevado a cabo una discriminación de la población, de acuerdo con su agrupación en núcleos de vida, hasta 2.000 habitantes, de 2.001 a $\mathbf{1 0 . 0 0 0}$ y de más de $\mathbf{1 0 . 0 0 0}$.

El rápido avance de casi todas las capitales y principales ciudades durante los últimos veinticinco años, en cuanto a urbanización, edificaciones, obras de mejoramiento y servicios de higiene, etc., se refiere, ha señalado y señala cada día con línea más fuerte, una clara separación de la población española según dichas condiciones, que pudiéramos llamar "urbanísticas», en que se desenvuelve.

Naturalmente, es muy difícil fijar límites de separación de esas zonas, mas el I. N. E., en esta primera información de los resultados 
(Peninsula e Islas adyacentes)

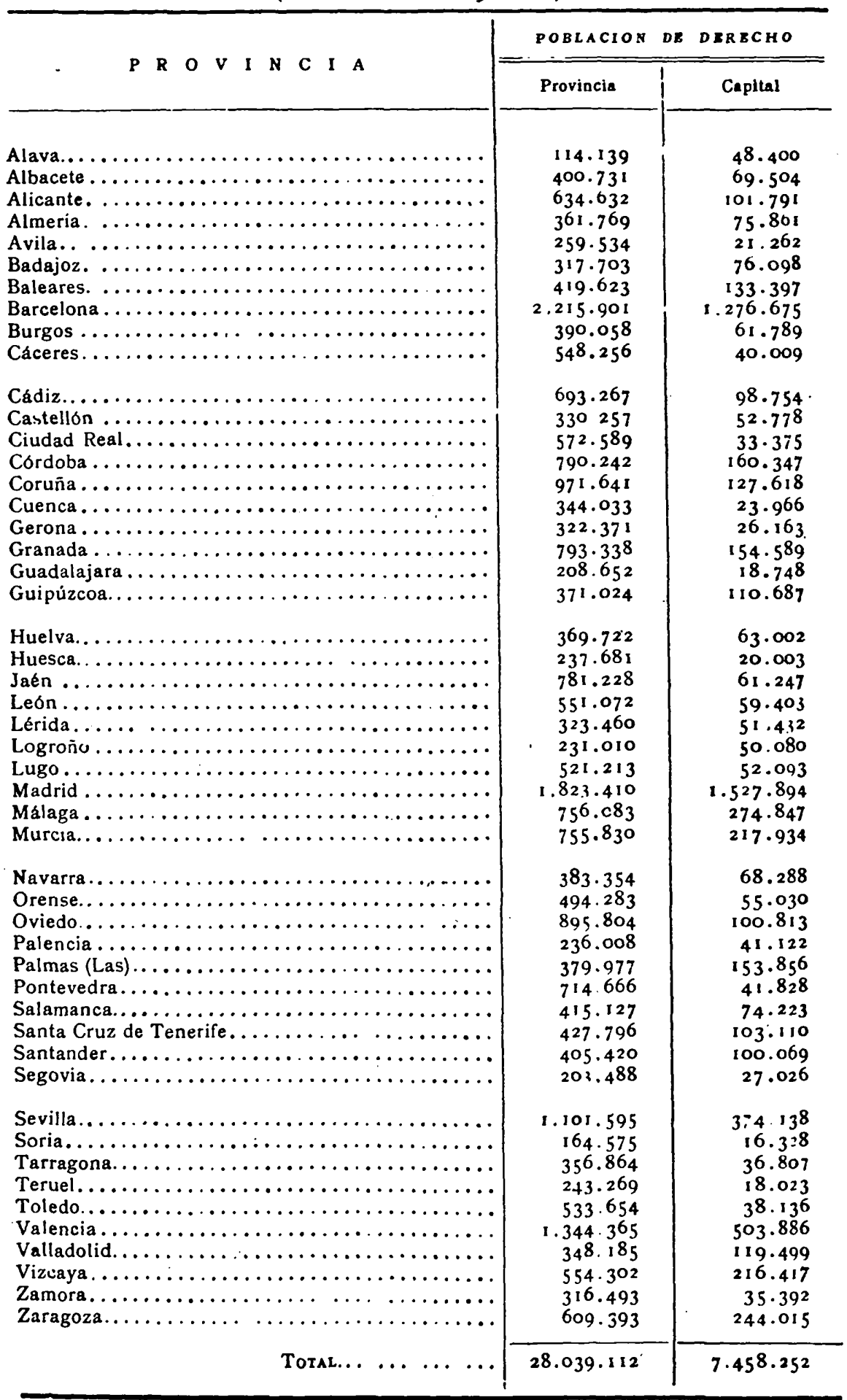


censales de 1950, distribuye la población en aquellos tres tipos de núcleos anteriormente citados, es decir, hasta 2.000 habitantes, masa calificada como rural, ya que en su totalidad asi lo es, de 2.001 a 10.000 , población que participa del ambiente rural, pero que también, $y$ en la mayoría de los núcleos en gran proporción, de las mejoras de servicios y obras municipales y que se la puede denominar semiurbana, $y$, por último, la población que vive en núcleos de más de 10.000 personas, población que, salvo raras excepciones, es típicamente urbana.

Existen, como es lógico, gradaciones dentro de los tres citados grupos.

En el cuadro II hemos establecido la comparación en cifras absolutas entre los seis censos del siglo, en la clasificación por grupos de población, en cuanto al número de Municipios y habitantes que absorben. Los datos se refieren a los Municipios de la Península e Islas adyacentes y afectan a la Población de Hecho (presentes y transeúntes), para que al hacer la comparación posterior con los núcleos ya citados, no falle la homogeneidad.

Agrupados en el cuadro III los datos del II en los tipos de población tantas veces repetidos, se observa la disminución de la población en los dos primeros grupos, a consecuencia del incremento demográfico principalmente, que varía el tipo de Municipio, a costa del aumento del 20 por 100 en los Municipios de más de $\mathbf{1 0 . 0 0 0 ~ h a b i t a n t e s . ~}$

Juzgamos muy interesante la comparación, Provincia a Provincia, de la distribución de la población por núcleos y por Municipios, para hacer resaltar la correlación directa o inversa entre la población de los Municipios y los núcleos de concentración de habitantes.

Precede a esa comparación el cuadro IV, en el que figuran distribuídos los Municipios españoles en los tres tipos adoptados.

La discrepancia se pone de manifiesto en las clásicas Provincias de gran dispersión demográfica en el territorio municipal, lo que hace que el Municipio correspondiente aparezca con una población encuadrada por su número en los grupos semiurbano o urbano.

Las Provincias gallegas, las cantábricas, junto con Albacete, Murcia, León. Canarias y bastantes andaluzas, son las que más destacan en esa variación de porcentajes. Mientras, por ejemplo, en Lugo, el 86.6 por 100 de la población vive en núcleos rurales, que no llegan a 2.000 habitantes; sólo tiene tres de esos Municipios cuya pob!ación representa únicamente el 1 por $\mathbf{1 0 0}$ del total, y el peso fuer:e del porcentaje se reparte entre los otros dos tipos de Municipios, con superioridad en el intermedio. 
C U A D R O I I

\begin{tabular}{|c|c|c|c|c|c|c|c|c|c|c|c|c|c|c|}
\hline \multirow{2}{*}{\multicolumn{3}{|c|}{$M U N I C I P I O S$}} & \multicolumn{2}{|c|}{1900} & \multicolumn{2}{|c|}{1910} & \multicolumn{2}{|c|}{1920} & \multicolumn{2}{|c|}{1930} & \multicolumn{2}{|c|}{$19 \div 0$} & \multicolumn{2}{|c|}{1950} \\
\hline & & & $\underset{\text { Núme- }}{\text { ró }}$ & Habitantes & $\underset{\text { ro }}{\text { Núme- }}$ & Habitantes & $\underset{\text { no }}{\text { Nume- }}$ & Habitailtes & $\underset{\text { No }}{\text { Núme- }}$ & Habitantes & $\underset{\text { Núme- }}{\text { Nú }}$ & Habitantes & $\underset{\text { ro }}{\text { Núme- }}$ & Habitantes \\
\hline $\mathrm{De}$ & menos de 101 hab & antes.... & 19 & 1.606 & 18 & 1.489 & 28 & 2.273 & $3 \mathrm{I}$ & 2.616 & 56 & $4 \cdot 498$ & 64 & $5 \cdot 377$ \\
\hline De & 1012500 & , $\ldots$. & $3 \cdot 176$ & 1.038 .001 & 3.042 & 995873 & 3.032 & 972.153 & 3.003 & 953.064 & 3.008 & 939.866 & 2.975 & 922.847 \\
\hline $\mathrm{De}$ & $501 \mathrm{a} \quad 1.000$ & 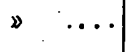 & $2 \cdot 367$ & $1.723 .53^{8}$ & $2.34^{\circ}$ & $1.691 \cdot 522$ & 2.243 & 1.598 .242 & 2.153 & 1.531 .634 & $2.15^{8}$ & 1.533 .892 & 2.077 & I. 472.892 \\
\hline $\mathrm{De}$ & 1.001 a 2.000 & • & I .654 & 2.362 .188 & $1.69 \mathrm{I}$ & 2.405 .223 & 1.699 & 2.390 .357 & 1.688 & 2.366 .501 & 1.623 & 2.298 .340 & 1.623 & 2.304 .616 \\
\hline De & $2.001 \mathrm{a} \quad 3.000$ & $"$ & 707 & 1.739 .040 & 716 & 1.764 .502 & 746 & 1.827 .678 & 745 & 1.818 .828 & 733 & 1.804 .467 & 732 & 1.793 .409 \\
\hline $\mathrm{De}$ & 3.001 a $\quad 5.000$ & $"$ & 671 & 2.604 .157 & 701 & 2.706 .047 & 700 & 2.689 .200 & 743 & 2.836 .325 & 727 & 2.811 .954 & 754 & 2.921 .020 \\
\hline De & 5.001 a 10.000 & $»$ & $45^{2}$ & 3.143 .699 & 497 & 3.430 .122 & 523 & 3.632 .657 & 577 & 4.018 .668 & $5^{67}$ & 3.922 .757 & 584 & 4.054 .930 \\
\hline De & 10.001 a 20.000 & - & 150 & $2.002 .68 \mathrm{I}$ & 178 & 2.403 .635 & I 94 & 2.646 .922 & 209 & 2.845 .905 & 245 & $3.268 .6 \mathrm{Ir}$ & 256 & $3 \cdot 360 \cdot 742$ \\
\hline De & 20.001 a 30.000 & • & 37 & 882.921 & 40 & 952.869 & 40 & 975.119 & 47 & 1.122 .133 & 61 & 1.457 .556 & 62 & $1.444 \cdot 739$ \\
\hline De & 30.001 a 50.000 & » & 15 & 563.503 & 16 & 586.497 & 23 & $787.61 \mathrm{~S}$ & 37 & 1.391 .325 & 38 & 1.488 .045 & 33 & 1.212 .766 \\
\hline De & 50.001 a 100.000 & $\nu$ & 12 & 856.723 & 14 & 934.850 & 18 & 1.213 .629 & 16 & 1.163 .036 & 20 & 1.376 .155 & 28 & 1.743 .076 \\
\hline De & 100.001 a 500.000 & $n$ & 4 & $603 \cdot 5 \times 3$ & 6 & $867 \cdot 303$ & 7 & 1.106 .083 & 9 & I. 555.435 & 16 & 2.802 .008 & 21 & 3.332 .672 \\
\hline & más de 500.000 & * & 2 & 1.072 .835 & 2 & I. 187.218 & 2 & 1.461 .231 & $i$ & 1.958 .397 & 2 & 2.169 .822 & 3 & $3.407 .68 y$ \\
\hline & Total. & & 9.266 & 18.594 .405 & $9.26 \mathrm{I}$ & 19.927 .155 & 9.254 & $21 \cdot 303 \cdot 162$ & 9.260 & $23.563 .8 \epsilon_{7}$ & 9.254 & 25.877 .974 & 9.212 & 27.976 .755 \\
\hline
\end{tabular}


. REVL-1952, núm. 64. GARCIA ALVAREZ, M.. ESTADISTICA. NUCLEOS DE AGRUPACION DE LA... 
CUADRO III

\begin{tabular}{|c|c|c|c|c|c|c|c|c|c|}
\hline \multirow{2}{*}{$M U N I C I P I O S$} & \multicolumn{3}{|c|}{$\begin{array}{llll}1 & 9 & 0 & 0\end{array}$} & \multicolumn{3}{|c|}{$\begin{array}{llll}1 & 9 & 1 & 0\end{array}$} & \multicolumn{3}{|c|}{$\begin{array}{llll}1 & 0 & 2 & 0\end{array}$} \\
\hline & $\underset{\text { rúmo- }}{\text { Nó }}$ & Hebitantos & $\begin{array}{l}\text { Tanto } \\
\text { Por } 100\end{array}$ & $\underset{\text { rume- }}{\text { Nún }}$ & Habitantos & $\begin{array}{c}\text { Tanto } \\
\text { Por } 100\end{array}$ & $\underset{\text { rome- }}{\text { Nume }}$ & Hebltapies & $\begin{array}{l}\text { Tanto } \\
\text { Por } 100\end{array}$ \\
\hline Hests 2.000 habitantes .................. & 7.216 & $5.125 \cdot 333$ & 27,6 & 7.091 & $5.094 \cdot 107$ & 25,6 & 7.001 & 4.973 .025 & 23,3 \\
\hline Do 2.000 \& 10.000 habitantes............... & 1.830 & 7.486 .896 & 40,3 & 1.914 & 7.900 .671 & 39,6 & 1.969 & 8.149 .535 & 38,3 \\
\hline De más de $10.000 .$. & 220 & 5.982 .176 & 32,1 & 256 & $6.932 \cdot 37^{2}$ & 34,8 & 284 & 8.190 .602 & 38,4 \\
\hline 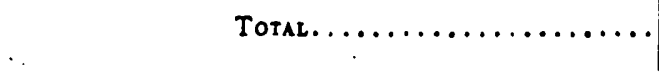 & 9.266 & 18.594 .405 & 100,0 & $9.26 !$ & 19.927 .150 & 100,0 & 9.255 & $21 \cdot 303 \cdot 162$ & 100,0 \\
\hline \multirow{2}{*}{$M U N I C I . P I O S$} & \multicolumn{3}{|c|}{$\begin{array}{llll}1 & 9 & 3 & 0\end{array}$} & \multicolumn{3}{|c|}{$\begin{array}{llll}1 & 9 & 4 & 0\end{array}$} & \multicolumn{3}{|c|}{$\begin{array}{llll}1 & 9 & 5 & 0\end{array}$} \\
\hline & $\underset{\text { ro }}{\text { Núme- }}$ & Habitantes & $\begin{array}{c}\text { Tanto } \\
\text { Por } 100\end{array}$ & $\underset{\text { ro }}{\text { Nume- }}$ & Habitantes & $\begin{array}{l}\text { Tanto } \\
\text { Por } 200\end{array}$ & $\underset{\text { ro }}{\text { Núme- }}$ & Habltantos & $\begin{array}{l}\text { Tanto } \\
\text { Por } 100\end{array}$ \\
\hline Hasta 2.000 habitantes .... & 6.875 & 4.853 .815 & 20,6 & 6.845 & 4.776 .596 & 18,5 & 6.739 & $4 \cdot 705 \cdot 712$ & 16,8 \\
\hline Do 2.000 a 10.000 habitantes........... & 2.065 & 8.673 .821 & 36,8 & 2.027 & $8 \cdot 539 \cdot 178$ & 33,0 & 2.070 & 8.769 .359 & 31,3 \\
\hline De más de 10.000 habitantes... & 320 & 10.036 .231 & 42,6 & 382 & $12 \cdot 562.197$ & 48,5 & 403 & 14.501 .684 & 51,9 \\
\hline Total........ & 9.260 & $33 \cdot 563.867$ & 100,0 & 9.254 & 25.877 .971 & 150,0 & 9.255 & 27.976 .755 & 100,0 \\
\hline
\end{tabular}




\section{UA D R I V}

\begin{tabular}{|c|c|c|c|}
\hline \multirow[b]{2}{*}{ PROVINCIA } & NUMERO & \multicolumn{2}{|c|}{ MUNICIPIOS } \\
\hline & Hasta 2000 hab. & $\begin{array}{c}\text { De } 2000 \text { a } 10000 \\
\text { habitantes }\end{array}$ & $\begin{array}{l}\text { De más } \\
\text { de } 10000 \text { hablt. }\end{array}$ \\
\hline 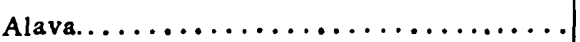 & 67 & 7 & $\boldsymbol{I}$ \\
\hline 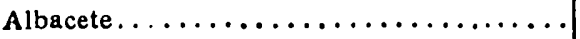 & $3 \mathbf{I}$ & 48 & 7 \\
\hline Alicante $\ldots \ldots \ldots \ldots \ldots \ldots \ldots \ldots \ldots$ & $8_{4}$ & 45 & 11 \\
\hline 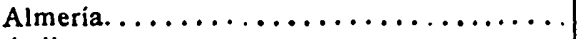 & 55 & 39 & 9 \\
\hline 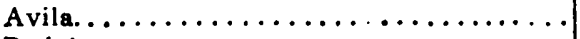 & 244 & 23 & 1 \\
\hline 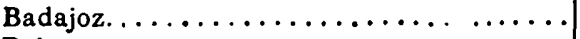 & 44 & 100 & 18 \\
\hline Baleares..$\ldots \ldots \ldots \ldots \ldots \ldots \ldots \ldots \ldots \ldots \ldots$ & 15 & $4 \mathrm{I}$ & 9 \\
\hline 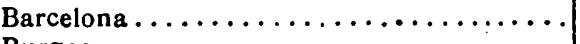 & 217 & 75 & 17 \\
\hline 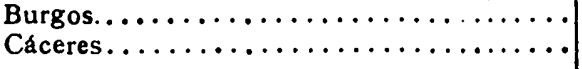 & $\begin{array}{l}479 \\
134\end{array}$ & $\begin{array}{l}22 \\
84\end{array}$ & $\begin{array}{l}3 \\
5\end{array}$ \\
\hline 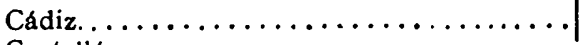 & 5 & 17 & 20 \\
\hline Castellon $\ldots \ldots \ldots \ldots \ldots \ldots \ldots \ldots$ & 100 & 36 & 5 \\
\hline Ciudad Real $\ldots \ldots \ldots \ldots \ldots \ldots \ldots \ldots$ & 34 & 49 & 15 \\
\hline Córdoba $\ldots \ldots \ldots \ldots \ldots \ldots \ldots \ldots \ldots$ & 9 & 45 & 21 \\
\hline Coruña $\ldots \ldots \ldots \ldots \ldots \ldots \ldots \ldots \ldots \ldots \ldots$ & I & $7 i$ & 22 \\
\hline 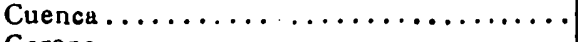 & 253 & 36 & I \\
\hline Gerona..$\ldots \ldots \ldots \ldots \ldots \ldots \ldots \ldots \ldots$ & 216 & 31 & 3 \\
\hline Granada $\ldots \ldots \ldots \ldots \ldots \ldots \ldots \ldots \ldots \ldots$ & 108 & 77 & 13 \\
\hline Guadalajara $\ldots \ldots \ldots \ldots \ldots \ldots \ldots \ldots \ldots$ & 398 & 7 & 1 \\
\hline Guipúzcoa........................ & $5^{\circ}$ & 29 & 8 \\
\hline 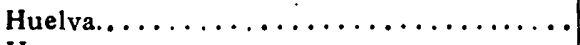 & 26 & 45 & 7 \\
\hline 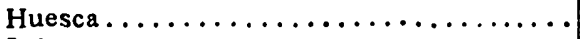 & 333 & 15 & $\mathbf{I}$ \\
\hline 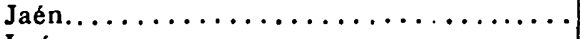 & 15 & 66 & 20 \\
\hline 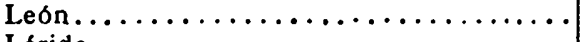 & 140 & 91 & 3 \\
\hline & 294 & 25 & 1 \\
\hline 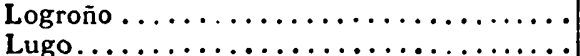 & $\begin{array}{r}163 \\
3\end{array}$ & 18 & 2 \\
\hline & $\begin{array}{r}3 \\
151\end{array}$ & $\begin{array}{l}51 \\
28\end{array}$ & 13 \\
\hline $\begin{array}{l}\text { Madrid } \ldots \ldots \cdots \\
\text { Málaga } \ldots \ldots \ldots \ldots \\
\end{array}$ & $\begin{array}{r}151 \\
33\end{array}$ & 28 & $\begin{array}{l}5 \\
0\end{array}$ \\
\hline 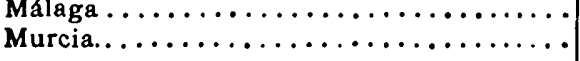 & $\begin{array}{r}33 \\
5\end{array}$ & $\begin{array}{l}58 \\
10\end{array}$ & $\begin{array}{r}9 \\
18\end{array}$ \\
\hline & 5 & I 9 & \\
\hline 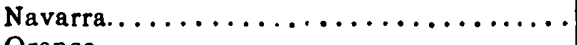 & 215 & 47 & 2 \\
\hline Orense $\ldots \ldots \ldots \ldots \ldots \ldots \ldots \ldots \ldots \ldots \ldots$ & 7 & 84 & 2 \\
\hline Oviedo $\ldots \ldots \ldots \ldots \ldots \ldots \ldots \ldots \ldots \ldots$ & 15 & 40 & 13 \\
\hline 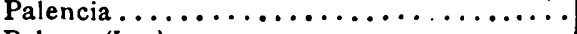 & 228 & 18 & 1 \\
\hline Palmas (Las) $\ldots \ldots \ldots \ldots \ldots \ldots \ldots \ldots \ldots$ & 7 & 23 & 5 \\
\hline 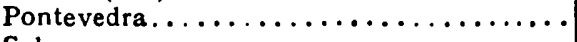 & 2 & 43 & 17 \\
\hline 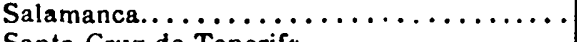 & 366 & 17 & 3 \\
\hline 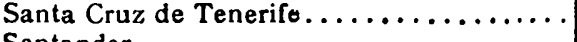 & 3 & 43 & 8 \\
\hline 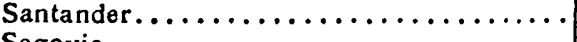 & 39 & 59 & 4 \\
\hline 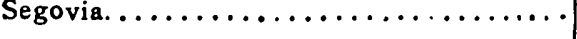 & 268 & 7 & $\mathbf{I}$ \\
\hline 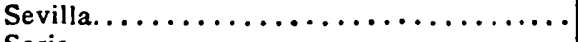 & I 5 & 65 & 22 \\
\hline 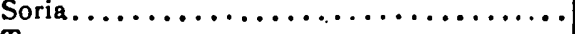 & 338 & 6 & $\mathbf{I}$ \\
\hline 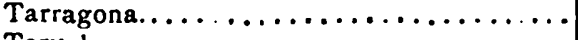 & 148 & 27 & 5 \\
\hline 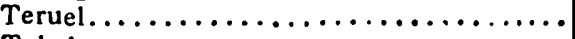 & 265 & 16 & I \\
\hline Toledo........................ & 120 & 80 & 6 \\
\hline 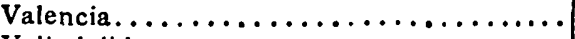 & 148 & 98 & 18 \\
\hline Valladolid...................... & 215 & 18 & 2 \\
\hline 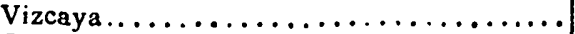 & 74 & 32 & 8 \\
\hline Zamora $\ldots \ldots \ldots \ldots \ldots \ldots \ldots \ldots \ldots \ldots$ & 293 & 12 & $\mathbf{2}$ \\
\hline Zaragoza..................... & 266 & 36 & 3 \\
\hline Tотат............... & 6.739 & 2.739 & 403 \\
\hline
\end{tabular}


En las Castillas y Aragón la correlación entre los núcleos y los Municipios es casi perfecta, mientras hay un tipo mixto en el que la discrepancia de porcentajes es ligera y variable, tipo al que corresponden Baleares y las Provincias de la zona mediterránea.

Es indudable que las condiciones de vida en las Provincias en que los Municipios abarcan una masa considerable de núcleos pequeños de población, tienen que ser mejores que en aquellas otras en que cada núcleo constituye un Municipio.

Estos Municipios son casi siempre económicamente débiles y es claro que no tienen medios a su alcance para acometer obras de mejoramiento de servicios municipales, como lo tendrían si el esfuerzo aunado de muchos núcleos cristalizase en la labor de un solo Ayuntamiento.

Esos 479 Municipios de Burgos, que no llegan a 2.000 habitantes, y de ellos un gran porcentaje ni a los 1.000 ; o los 398 de Guadalajara, 366 de Salamanca, 338 de Soria, 333 de Huesca, etc., fusionados en un número mucho menor, podrían proporcionar a sus habitantes un mejoramiento notable en sus servicios municipales, con la ventaja, además, de que el núcleo principal de los agrupados, en el que residiria el Ayuntamiento, sería siempre el punto de atracción de los restantes, en cuanto a la resolución de pequeños problemas diarios, que hoy exigen un desplazamiento lejano hasta Municipios de mayor categoria.

No hemos hecho más que traducir a números la realidad de la gran variabilidad en la densidad demográfica de los Municipios, de unas a otras regiones españolas, y esbozar una posible solución.

Naturalmente, son los Organismos que dirigen el engranaje de la vida local española los encargados de sacar conclusiones de esas cifras, que son la fotografía de la situación topo-demográfica de la población española al finalizar el año $\mathbf{1 9 5 0 .}$

M. Garcia Alvarez 